\title{
Remark on coefficient fields in complete local rings
}

\author{
By \\ J. N. Mordeson \\ (Communicated by Professor M. Nagata, April 20, 1965)
}

Let $R^{\prime} \supseteq R$ be complete local rings (not necessarily Noetherian) of prime characteristic $p$ such that $R^{\prime}$ dominates $R$. Denote the residue class fields of $R^{\prime}$ and $R$ by $K^{\prime}$ and $K$, respectively. Let $g$ be the natural homomorphism of $R^{\prime}$ onto $K^{\prime}$.

In $\left[2\right.$, p. 92], it is proved that under the assumption $R^{\prime p^{n}} \subseteq R$ for some positve integer $n$, there exists a coefficient field of $R$ which is extendable to one of $R^{\prime}$ if (1) $g\left(R^{\prime p i} \cap R\right)=K^{\prime p^{i}} \cap K(i=1,2, \cdots)$ and (2) there exists a $p$-basis $B$ of $K^{\prime}$ such that $K^{\prime p^{*}}\left(C^{\prime}\right)=K$ where $C=\left\{b^{p *} \mid b \in B, e=e(b)\right.$ is the exponent of $b$ over $\left.K\right\}$.

Our purpose is to note the following two extensions of this result for $K^{\prime}$ pure inseparable and of arbitrary exponent over $K$. Neither depends on $R^{\prime}$ being integral over $R$.

Theorem 1. Suppose $\left.\bigcap_{i}^{\infty} R^{\prime p^{i}} \mid R\right]=R$. If (1) $g\left(R^{\prime p^{i}} \cap R\right)=$ $K^{\prime p^{i}} \cup K(i=1,2, \cdots)$ and (2) there exists a $p$-basis $B$ of $K^{\prime}$ such that $K^{\prime p^{i}}(C) \supseteq K(i=1,2, \cdots)$, then there exists a coefficient field of $R$ which is extendable to one of $R^{\prime}$.

The assumption $\bigcap_{i}^{\infty} R^{\prime p^{i}}[R]=R$ implies the maximal perfect subfield $P=\bigcap_{i}^{\infty} K^{\prime p i}$ of $K^{\prime}$ is contained in $K$. The following considers the case $P \supseteq P \cap K$. Let $P^{\prime}=\bigcap_{i}^{\infty} R^{\prime p^{i}}$.

Theorem 2. Suppose $g\left(P^{\prime} \cap R\right)=P \cap K$. If (1) of Theorem 1 holds and (2) there exists a set $I) \subseteq P \cap K$ and a $p$-basis $B$ of $K^{\prime}$ 
such that $K^{p}(D, C)=K$, then there exists a coefficient field of $R$ which is extendable to one of $R^{\prime}$.

That these two theorems extend the result in [2, p. 92] is a consequence of the following: $K^{\prime n}(C)=K$ implies $K^{\prime n n+1}(C)=K^{p}(C)$ and since $K^{\prime p n}=K^{\prime p n+1}\left(B^{p n}\right) \subseteq K^{\prime p^{n+1}}(C)$, we have $K^{p}(C)=K$. For Theorem $1, K^{p}(C)=K$ implies $K^{p i}(C)=K$ whence $K^{\prime p i}(C) \supseteq K(i=1$, $2, \cdots)$. Clearly, $R^{\prime p n} \subseteq R$ implies $\bigcap_{i}^{\infty} R^{\prime p i}[R]=R$. For Theorem 2, we can take $D$ to be the empty set and clearly $K^{\prime{ }^{n}} \subseteq K$ implies $g\left(P^{\prime} \cap R\right)$ $=P \cap K$ since $g \bigcap_{i}^{\infty} R^{p i}=P \subseteq K$ here. Thus, by Theorem 2, the assumption $R^{\prime p n} \subseteq R$ in [2] can be replaced by $K^{\prime p n} \subseteq K$ whence $R^{\prime}$ need not be integral over $R$.

Proof of Theorem 1. By (1) there exists a set of representatives $B_{0}$ of $B$ in $R^{\prime}$ such that for all $b \in B, b^{b^{\circ}} \in K$ implies $b_{0}^{b_{0}} \in R$ where $b_{0} \in B_{0}$ and $g b_{0}=b$. By the existence lemma in [2, p. 91], there exists a coefficient field $k^{\prime}$ of $R^{\prime}$ containing $B_{0}$. Clearly, $k^{\prime}$ contains a set of representatives $C_{0} \subseteq R$ of $C$ by the choice of $B_{0}$. By (2), $g k^{\prime p i}\left(C_{0}\right)$ $\supseteq K$. Thus, there exists a field $k \subseteq \bigcap_{i}^{\infty} k^{\prime p i}\left(C_{0}\right)$ such that $g k=K$. Since $C_{0} \subseteq R$ and $\bigcap_{i}^{\infty} R^{\prime p^{i}}[R]=R, k \subseteq R$. Q.E.D.

Proof of Theorem 2. By hypothesis and (1), there exists a set of representatives $B_{0}$ of $B$ in $R^{\prime}$ such that $R^{\prime p i}\left[B_{0}\right](i=1,2, \cdots)$ contain sets of represenatives $D_{0}, C_{0} \subseteq R$ of $D, C$ respectively. By (2), there exists a subset $G \subseteq D \cup C$ which is a $p$-basis of $K$. Hence, $R^{\prime p^{i}}\left[B_{0}\right]$ contains a set of representatives $G_{0} \subseteq R$ of $G$. The remainder of the proof follows as in the proof of Theorem 27, [3, p, 306], from the inclusion $R^{\prime p i}\left[B_{0}\right] \supseteq R^{p i}\left[G_{0}\right]$ and from the completeness of $R^{\prime}$ and R. Q.E.D.

If $K$ is separable algebraic over $(P \cap K)(C)$, then (2) of Theorem 2 holds. It follows that $B$ is an algebraically independent set over $P$ and thus over $P \cap K$. Hence $C$ is an algebraically independent set over $P \cap K$. By footnote 14, [1, p. 378], $D \cup C$ is a $p$-basis of $(P \cap K)(C)$ where $D$ is any $p$-basis of $P \cap K$. Since $K$ is separable 
algebraic over $(P \cap K)(C), D \cup C$ is a $p$-basis of $K$ by footnote 14 or Theorem 8, [1].

\section{REFERENCES}

[1] S. MacLane, "Modular fields, I." Duke Math. J. 5 (1939), pp. 372-393.

[2] M. Nagata, "Note on coefficient fields of complete local rings," Mem. Coll. Sci. Univ. Kyoto 32 (1959-1960), pp. 91-92.

[3] O. Zariski and P. Samuel, "Commutative Algebra," vol. II, D. Van Nostrand, Princeton, N. J., 1960.

Creighton University 